\title{
Enterocolitis in Patients with Cancer Treated with Docetaxel
}

\author{
FRÉDÉRIC FITENI ${ }^{1,2}$, MARIE-JUSTINE PAILLARD ${ }^{3,4}$, EMELINE ORILLARD $^{3}$, LORIANE LEFEBVRE $^{3}$, \\ SARAH NADJAFIZADEH ${ }^{3}$, ZOHAIR SELMANI ${ }^{3}$, SALIM BENHAMIDA ${ }^{3}$, ANTOINE ROLAND $^{3}$, \\ ANTOINE BAUMANN ${ }^{3}$, ANGÉLIQUE VIENOT ${ }^{4,5}$, NADINE HOUÉDÉ ${ }^{1}$ and XAVIER PIVOT ${ }^{3}$ \\ ${ }^{1}$ Department of Medical Oncology, University Hospital of Nîmes, Nîmes, France; \\ ${ }^{2}$ Biometrics Unit, Cancer Institute of Montpellier, Montpellier, France; \\ ${ }^{3}$ Department of Medical Oncology, ${ }^{4}$ Methodology and Quality of Life in Oncology Unit, INSERM UMR 1098, and \\ ${ }^{5}$ Department of Gastroenterology, University Hospital of Besançon, Besançon, France
}

\begin{abstract}
Background: Enterocolitis is a rare, but serious gastrointestinal complication associated with docetaxel-based chemotherapy in patients with cancer. The incidence, clinical presentation and outcome of enterocolitis in patients with cancer treated with docetaxel-based chemotherapy was assessed in this study Patients and Methods: All patients treated with docetaxel for cancer between January 2010 and December 2014 at the University Hospital of Besançon were identified and their medical records reviewed. Results: During this period, 1,227 patients received docetaxel chemotherapy and gastrointestinal events occurred in 381 (31.1\%) patients. In multivariate analysis, a higher risk of gastrointestinal events was associated with a higher dose of docetaxel $\left(\geq 75 \mathrm{mg} / \mathrm{m}^{2}\right)$ $($ odds $\operatorname{ratio}(O R)=46.2 ; 95 \%$ confidence interval $(C I)=5.4$ 397.0, $p=0.0005)$ and the first cycle of docetaxel $(O R=4.2$; 95\% CI=1.8-10.1, $p=0.001$ ). Among the 381 patients with gastrointestinal events, grade 3/4 neutropenia, diarrhea, febrile neutropenia, mucositis, nausea/vomiting, and rectal bleeding were diagnosed in 65 (17.1\%), 51 (13.4\%), 37 (9.7\%); 12 $(3.1 \%)$, seven $(1.8 \%)$ and three $(0.8 \%)$ patients, respectively; 54 patients (14.2\%) were hospitalized. Computed tomographic scan was performed for 39 patients (10.2\%). Twenty-seven patients presented radiological signs of enterocolitis. Three deaths $(0.8 \%)$ related to enterocolitis were recorded. Docetaxel was resumed in 261 patients (68.5\%) and the dose was reduced in 89 patients (23.4\%). Docetaxel was discontinued in 120 patients (31.5\%). Conclusion: Gastrointestinal events in patients treated with docetaxel may be a potential sign of fatal enterocolitis and require particular attention. Dose reduction at the first cycle may reduce the risk of such events.
\end{abstract}

Correspondence to: Frédéric Fiteni, MD, Ph.D., Medical Oncology Department, Nimes University Hospital, Rue du Pr Henri Pujol 30029 Nîmes Cedex 9, France. Tel: +33 4466683301, e-mail: fredericfiteni@gmail.com

Key Words: Enterocolitis, typhlitis, docetaxel, cancer, chemotherapy.
Enterocolitis is defined as an inflammation of the gastrointestinal (GI) tract. Enterocolitis in patients with cancer was first described as a serious complication of hematological cancer with severe myelosuppression (1). The terms 'neutropenic enterocolitis' (2), 'necrotizing enterocolitis' (3), 'iliocecal syndrome' (4) and 'typhlitis' (5) have been used synonymously by authors (typhlitis comes from the Greek typhlon, meaning cecum). Patients receiving chemotherapy with enterocolitis present symptoms of acute abdominal pain with possibly associated neutropenia, fever, or diarrhea (with or without blood). Pathological alterations have been described, including edema of the mucosa or entire intestinal wall, mucosal ulceration, focal hemorrhage and mucosal or transmural necrosis (5). Enterocolitis associated with docetaxel was first described by Cardenal et al. in 1996 (6). In 2004, Li et al. identified 1,350 patients treated with taxane-based chemotherapy (paclitaxel or docetaxel) for breast cancer. Among these patients, they focused on those who were admitted to hospital for GI complications: 64 were hospitalized and 14 patients were diagnosed with colitis (7). Nevertheless, many patients treated with docetaxel present GI symptoms but are not necessarily hospitalized. Moreover, docetaxel is prescribed for many different types of cancer. Therefore, it is important to know the frequency of gastrointestinal events with docetaxel to improve the diagnosis of enterocolitis and its management. The aim of our study was to review the medical records of patients treated with docetaxel at the University Hospital of Besançon to assess the frequency, clinical presentation and clinical outcome of enterocolitis associated with docetaxel.

\section{Patients and Methods}

Study population. We performed a search using computerized software for chemotherapy prescriptions, called BPC (acronym for Bonne Pratique de Chimiothérapie) which was implemented for routine use at the University Hospital of Besançon. Through this database, all patients treated with docetaxel between January 2010 and December 2014 were identified. We reviewed the patients' 
medical records and documented their age, sex, type of cancer, disease stage, dose and schedule of docetaxel, GI events (defined by acute abdominal pain or diarrhea during their treatment with docetaxel), presenting symptoms of GI events (using the use the International Common Terminology Criteria for Adverse Events (CTCAE), version 4.0, for adverse event reporting (8)) clinical management and outcome of GI events.

Statistical analysis. We used means and range for continuous variables, and proportions for qualitative variables. We compared proportions using chi-squared test, or Fisher's exact test when appropriate. Univariate and multivariate logistic regression were performed to identify the variables associated with GI events. A $p$ value of 0.05 or lower was considered statistically significant. All analyses were performed using SAS software, Version 9.3 (SAS Institute, Cary NC, USA).

\section{Results}

Between January 2010 and December 2014, 1227 patients received docetaxel chemotherapy at the University Hospital of Besançon. Among these patients, 679, 221, 151, 96, 58, and 22 patients had breast, lung, prostate, head and neck, GI and other cancer, respectively. The characteristics of patients are presented in Table I.

GI events occurred in 381 (31.1\%) patients. The mean time to onset of GI events was 5.4 days after the administration of chemotherapy. Among the 381 patients, GI events occurred after receiving the first cycle of docetaxel in 258 cases $(67.7 \%)$, after the second cycle in 63 patients (16.5\%), after the third cycle in 21 patients (5.8\%) and after the fourth cycle or more in 41 patients $(10.8 \%)$. In multivariate analysis, a higher risk of GI events was associated with a higher dose of docetaxel $\quad\left(\geq 75 \quad \mathrm{mg} / \mathrm{m}^{2}\right) \quad$ (odds $\quad \operatorname{ratio}(\mathrm{OR})=46.2 ; 95 \%$ confidence interval $(\mathrm{CI})=5.4-397.0, p=0.0005)$ and the first cycle of docetaxel (odds ratio $=4.2 ; 95 \%$ confidence interval $=1.8-10.0, p=0.001$ ) (Table II).

Among the 381 patients with GI events, diarrhea, mucositis, acute abdominal pain, neutropenia, fever, nausea/vomiting, febrile neutropenia and rectal bleeding manifested in 253 (66.4.2\%), 141 (37\%), 125 (32.8\%), 81 (21.3\%), $75(19.7 \%), 62(16.3 \%), 40(10.5 \%)$ and $17(4.5 \%)$ patients, respectively. Grade 3/4 neutropenia, diarrhea, febrile neutropenia, mucositis, nausea/vomiting, and rectal bleeding were diagnosed in $65(17.1 \%), 51(13.4 \%), 37$ $(9.7 \%) ; 12(3.1 \%)$, seven $(1.8 \%)$ and three $(0.8 \%)$ patients, respectively.

Fifty-four patients $(14.2 \%)$ were hospitalized. Computed tomographic (CT) scan was performed for 39 patients $(10.2 \%)$. Twenty-seven patients presented radiological signs of enterocolitis: diffuse or localized thickening of the colonic wall was observed in 24 patients, pneumoperitoneum was evident in two patients and one patient presented diffuse thickening of the colon associated with peritoneal effusion and dilatation of the colon.
Table I. Characteristics of the patients included in this study.

\begin{tabular}{|c|c|}
\hline Characteristic & All patients $(n=1227)$ \\
\hline Median age (range), years & $59.9(20.8-95.2)$ \\
\hline \multicolumn{2}{|l|}{ Gender, $\mathrm{n}(\%)$} \\
\hline Male & $451(36.7 \%)$ \\
\hline Female & $776(63.2 \%)$ \\
\hline \multicolumn{2}{|l|}{ Performance status, n (\%) } \\
\hline 0 & $472(38.5 \%)$ \\
\hline $1 / 2$ & $332(27.1 \%)$ \\
\hline $3 / 4$ & $36(29.3 \%)$ \\
\hline Missing data & $387(31.5 \%)$ \\
\hline \multicolumn{2}{|l|}{ Type of disease, $\mathrm{n}(\%)$} \\
\hline Breast cancer & $502(40.9 \%)$ \\
\hline Lung cancer & $208(17.0 \%)$ \\
\hline Prostate cancer & $149(12.1 \%)$ \\
\hline Head and neck cancer & $90(7.3 \%)$ \\
\hline Gastroesophageal cancer & $35(2.9 \%)$ \\
\hline Anal cancer & $12(1.0 \%)$ \\
\hline Other & $38(3.1 \%)$ \\
\hline Missing data & $193(15.7 \%)$ \\
\hline \multicolumn{2}{|l|}{ Setting, n (\%) } \\
\hline Neoadjuvant & $216(17.6 \%)$ \\
\hline Adjuvant & $335(27.3 \%)$ \\
\hline Metastatic & $475(38.7 \%)$ \\
\hline Missing data & $203(16.5 \%)$ \\
\hline \multicolumn{2}{|c|}{ Previously exposed to taxane, $\mathrm{n}(\%)$} \\
\hline Yes & $920(75.0 \%)$ \\
\hline No & $106(8.6 \%)$ \\
\hline Missing data & $201(16.4 \%)$ \\
\hline \multicolumn{2}{|l|}{ Docetaxel dose, n (\%) } \\
\hline$\leq 75 \mathrm{mg} / \mathrm{m}^{2}$ & $787(64.1 \%)$ \\
\hline$>75 \mathrm{mg} / \mathrm{m}^{2}$ & $246(20.0 \%)$ \\
\hline Missing data & $194(15.8 \%)$ \\
\hline \multicolumn{2}{|l|}{ Docetaxel regimen, n (\%) } \\
\hline Monotherapy & $682(55.6 \%)$ \\
\hline + Chemotherapy & $393(32.0 \%)$ \\
\hline + Antibody & $152(12.4 \%)$ \\
\hline
\end{tabular}

Seventy-four patients (19.4\%) received broad-spectrum antibiotics. Six patients (1.6\%) presented septic shock during hospitalization. Three patients $(0.8 \%)$ underwent surgery: the two with pneumoperitoneum and the patient with peritoneal effusion and dilatation of colon on the CT scan. Three deaths $(0.8 \%)$ related to enterocolitis were recorded.

Docetaxel was resumed in 261 patients $(68.5 \%)$ and the dose was reduced in 89 (23.4\%) patients. Docetaxel was discontinued in 120 patients $(31.5 \%)$. Docetaxel was replaced by paclitaxel in 33 patients $(8.7 \%)$.

\section{Discussion}

To our knowledge, our study is the first to analyze clinical presentation and treatment of enterocolitis with docetaxelbased chemotherapy regardless of cancer type. The mechanism of enterocolitis as a complication of taxane-based 
Table II. Patients characteristics associated with gastrointestinal events.

\begin{tabular}{|c|c|c|c|c|c|c|}
\hline \multirow[t]{2}{*}{ Characteristic } & \multicolumn{2}{|c|}{ Gastrointestinal events, n (\%) } & \multicolumn{2}{|c|}{ Univariate analysis } & \multicolumn{2}{|c|}{ Multivariate analysis } \\
\hline & No $(n=741)$ & Yes $(n=381)$ & OR $(95 \% \mathrm{CI})$ & $p$-Value & OR $(95 \% \mathrm{CI})$ & $p$-Value \\
\hline Median age, years & & & & 0.93 & & \\
\hline$<59.9$ & $395(66.2 \%)$ & $202(33.8 \%)$ & 1 & & & \\
\hline$\geq 59.9$ & $346(65.9 \%)$ & $179(34.1 \%)$ & $1.01(0.8-1.3)$ & & & \\
\hline Gender & & & & $<0.0001$ & & 0.1 \\
\hline Male & $335(78.6 \%)$ & $91(21.4 \%)$ & 1 & & 1 & \\
\hline Female & $406(58.3 \%)$ & $290(41.7 \%)$ & $2.6(2.0-3.5)$ & & $4.0(0.8-20.7)$ & \\
\hline Performance status & & & & 0.0004 & & 0.97 \\
\hline $0 / 1$ & $437(62.9 \%)$ & $253(36.4 \%)$ & 1 & & 1 & \\
\hline $2 / 3 / 4$ & $107(79.3 \%)$ & $28(20.7 \%)$ & $0.5(0.3-0.7)$ & & $1.0(0.2-4.4)$ & \\
\hline Setting & & & & $<0.0001$ & & 0.84 \\
\hline (Neo)adjuvant & $251(46.8 \%)$ & $285(53.2 \%)$ & $4.5(3.4-5.9)$ & & 1 & \\
\hline Metastatic & $368(79.8 \%)$ & $93(20.2 \%)$ & 1 & & $0.9(0.3-2.7)$ & \\
\hline Previously exposed to taxane & & & & 0.028 & & 0.43 \\
\hline No & $542(60.6 \%)$ & $352(39.4 \%)$ & $2.0(1.3-3.2)$ & & 1 & \\
\hline Yes & $78(75.7 \%)$ & $25(24.3 \%)$ & 1 & & $0.5(0.1-2.6)$ & \\
\hline Docetaxel dose & & & & $<0.0001$ & & 0.0005 \\
\hline$<75 \mathrm{mg} / \mathrm{m}^{2}$ & $117(86.7 \%)$ & $18(13.3 \%)$ & 1 & & 1 & \\
\hline$\geq 75 \mathrm{mg} / \mathrm{m}^{2}$ & $500(58.1 \%)$ & $361(41.9 \%)$ & $2.5(1.9-3.4)$ & & $46.2(5.4-397.0)$ & \\
\hline Cycle of event & & & & $<0.0001$ & & 0.001 \\
\hline First cycle & $24(8.5 \%)$ & $258(91.5 \%)$ & $7.5(4.4-12.6)$ & & $4.2(1.8-10.0)$ & \\
\hline After first cycle & $68(41.0 \%)$ & $98(59.0 \%)$ & 1 & & 1 & \\
\hline Docetaxel regimen & & & & 0.03 & & \\
\hline Monotherapy & $354(66.5 \%)$ & $178(33.5 \%)$ & 1 & & 1 & \\
\hline + Chemotherapy & $76(61.3 \%)$ & $48(38.7 \%)$ & $1.3(0.8-1.9)$ & & $0.4(0.1-1.0)$ & 0.10 \\
\hline+ Antibody & $143(57.0 \%)$ & $108(43.0 \%)$ & $1.5(1.1-2.0)$ & & $1.4(0.5-4.1)$ & 0.6 \\
\hline
\end{tabular}

OR: Odds ratio; CI: confidence interval.

chemotherapy is still not well known. The clinical presentation of enterocolitis is variable and there is no clear definition. Nesher and Rolston tried to define major diagnostic criteria: neutropenia, bowel wall thickening $(>0.4 \mathrm{~cm}$ in transverse scan in any segment of the bowel for at least $0.3 \mathrm{~cm}$ length in longitudinal scan) and fever; and minor criteria: abdominal pain, abdominal distention, abdominal cramping, diarrhea and bleeding in the lower gastrointestinal tract (9). The clinical presentation is typically associated with diarrhea or abdominal pain $(5,7,10,11)$; therefore, we selected patients who presented acute abdominal pain or diarrhea during their treatment with docetaxel. We showed that these GI events occurred frequently (31.1\% of patients) and we observed three deaths related to enterocolitis. In 2017, the National Cancer Institute, in collaboration with the National Agency for the Safety of Medicines and Health Products in France revealed five cases of death between August 2016 and February 2017 related to enterocolitis in patients treated with docetaxel for adjuvant breast cancer therapy and provided precautions regarding the use of docetaxel (12).

Most of the papers published on enterocolitis in patients with solid tumours are based on case reports $(7,10)$ and there is only one cohort on patients treated with taxane-based chemotherapy, published by Li et al. in 2004 (7). In their study, they included only patients with breast cancer and identified patients who were admitted with GI complications after taxane-based chemotherapy (paclitaxel or docetaxel). In our study, 24 patients of 1,227 patients (2.0\%) had radiological signs of enterocolitis, which is consistent with the results of Li et al. where 10 out of 520 patients $(1.9 \%)$ who received docetaxel treatment developed enterocolitis (the authors did not specify what criteria were used for the diagnosis of enterocolitis).

In our study, enterocolitis appears to have been related to a higher dose of docetaxel $\left(\geq 75 \mathrm{mg} / \mathrm{m}^{2}\right)$ and the first cycle of docetaxel. Reduction of docetaxel dose at the first cycle may successfully prevent enterocolitis, with a dose increase from the second cycle if no enterocolitis occurs. Dose adaptation needs to be balanced with efficiency, especially if there is a clinical indication for a more rapidly efficacious therapy. In the cohort of Li et al., the authors did not find any significant differences in age, doses, and number of treatment cycles among patients who tolerated chemotherapy without complications, patients who required admission for 
nonenterocolitis gastrointestinal complications, and patients admitted with enterocolitis (7).

There have been no randomized trials on the treatment of enterocolitis, which can be through both medical and surgical intervention. Urbach et al. proposed indications for surgery: pneumoperitoneum, peritonitis, shock, uncontrolled hemorrhage, and clinical deterioration after medical treatment (5). The medical treatment is based on administration of intravenous fluids and intravenous antibiotics, elimination of oral intake, and correction of cytopenia and coagulopathy. The antibiotic regimen should cover Gram-negative organisms, the enterococci and anaerobic bacteria (13). However, the decision whether or not a patient presenting GI symptoms after a docetaxel injection needs hospitalization is not easy for physicians. Therefore, further investigations are warranted to provide guidelines regarding the management of enterocolitis after docetaxel administration.

In cases of enterocolitis, docetaxel can be replaced by paclitaxel. Nevertheless, caution is necessary because paclitaxel can also cause enterocolitis. However, the risk is lower with paclitaxel; Li et al. showed that the risk ratio of developing enterocolitis after paclitaxel versus docetaxel therapy was 0.35 (7). In cases of non-severe GI symptoms, docetaxel can be resumed with a reduced dose and a close monitoring of the patient. Nevertheless, the criteria for defining non-severe GI events are not standardized and further research is necessary to define these criteria and help physicians in their decision-making.

In summary, out of 1,227 patients with cancer treated with docetaxel, grade 3/4 GI events were rare, with radiological signs of enterocolitis being found in 27 patients, but can be fatal. Enterocolitis seems to be associated with the dose of docetaxel and the first cycle of therapy. Further research is necessary to clearly define enterocolitis in this setting and provide guidelines regarding the management of such patients.

\section{Conflicts of Interest}

The Authors declare that they have no conflict of interest in regard to this study.

\section{References}

1 Shamberger RC, Weinstein HJ, Delorey MJ and Levey RH: The medical and surgical management of typhlitis in children with acute nonlymphocytic (myelogenous) leukemia. Cancer 57(3): 603-609, 1986.

2 Wade DS, Nava HR and Douglass HO: Neutropenic enterocolitis. Clinical diagnosis and treatment. Cancer 69(1): 1723, 1992.

3 Amromin GD and Solomon RD: Necrotizing enteropathy: a complication of treated leukemia or lymphoma patients. JAMA 182: 23-29, 1962.

4 Sherman NJ and Woolley MM: The ileocecal syndrome in acute childhood leukemia. Arch Surg 107(1): 39-42, 1973.

5 Urbach DR and Rotstein OD: Typhlitis. Can J Surg 42(6): 415419, 1999.

6 Cardenal F, Montes A, Llort G, Seguí J and Mesía R: Typhlitis associated with docetaxel treatment. J Natl Cancer Inst 88(15): 1078-1079, 1996.

7 Li Z, Ibrahim NK, Wathen JK, Wang M, Menchu RPM, Valero V, Theriault A, Buzdar U and Hotobagyi GN: Colitis in patients with breast carcinoma treated with taxane-based chemotherapy. Cancer 101(7): 1508-1513, 2004.

8 National Institute of Cancer: Common Terminology Criteria for Adverse Events (CTCAE). NIH Publ 2009: 1-71, 2010.

9 Nesher L and Rolston KVI: Neutropenic enterocolitis, a growing concern in the era of widespread use of aggressive chemotherapy. Clin Infect Dis 56(5): 711-717, 2013.

10 Oehadian A and Fadjari TH: Neutropenic enterocolitis in breast cancer patient after taxane-containing chemotherapy. Acta Med Indones 40(1): 29-33, 2008.

11 Wade DS, Nava HR and Douglass HO: Neutropenic enterocolitis. Clinical diagnosis and treatment. Cancer 69(1): 1723, 1992.

12 Sous IT, De A, Confr C, Europ A, Ag BP, Alsympca III and Cancer SP: Lettre aux professionnels de santé, 2014.

13 Portugal R and Nucci M: Typhlitis (neutropenic enterocolitis) in patients with acute leukemia: a review. Expert Rev Hematol 10(2): 169-174, 2017. 